\title{
EFECTO DE LA INCLUSIÓN DE DIFERENTES FUENTES LIPÍDICAS EN LA DIETA DE ENGORDE SOBRE EL PERFIL DE ÁCIDOS GRASOS POLIINSATURADOS EN FILETE DE PACO Piaractus brachypomus
}

Wilfredo Vásquez Quispesivana*a, Raúl Porturas Olaechea ${ }^{a}$, Fredy Crispín Sánchez $^{\mathrm{a}}$

\section{RESUMEN}

La investigación tuvo como objetivo evaluar el efecto de la inclusión de diferentes fuentes lipídicas en la dieta de engorde sobre el perfil de ácidos grasos poliinsaturados AGPI en los filetes de Piaractus brachypomus. Se utilizó cuatro tipos de aceite; palma, maíz, soja y pescado, para elaborar dietas cumpliendo los requerimientos nutricionales de la especie. Se alimentaron durante dos meses con estas cuatro dietas y se utilizó como control un alimento comercial. Se determinó el perfil de ácidos grasos en los aceites, en las dietas y en los filetes de Piaractus brachypomus antes y después del experimento. Los niveles de AGPI omega-6 como el ácido araquidónico (AA) y los AGPI omega-3 como el ácido eicosapentaenoico (EPA) y el docosahexaenoico (DHA) fueron analizados. La dieta elaborada con aceite de pescado obtuvo el mejor perfil de AGPI en los filetes (2,11\%; 4,64 \% y 7,0 \% de AA; EPA y DHA, respectivamente), sin embargo, la fuente lipídica de origen vegetal, que mejor influyó en el incremento de ácidos grasos omega-3 en filete de Piaractus brachypomus $(3,11 \% ; 0,82$ $\%$ y $3,71 \%$ de AA, EPA y DHA) fue el aceite de palma. Se demostró la capacidad de mejorar el perfil de AGPI en filetes de Piaractus brachypomus.

Palabras clave: Piaractus brachypomus, ácidos grasos, filete, palma, maíz, soja, pescado, alimento.

\section{EFFECT OF THE INCLUSION OF DIFFERENT LIPID SOURCES IN THE DIET ON THE POLYUNSATURATED FATTY ACIDS PROFILE IN Piaractus brachypomus FILLET}

\begin{abstract}
This research evaluated the effect of the inclusion of different lipid sources in the diet on the on the polyunsaturated fatty acids profile PUFA in Piaractus brachypomus fillet. Four types of oil were used; palm, corn, soy and fish oil, to formulate diets that comply with

\footnotetext{
${ }^{a}$ Facultad de Pesquería, Universidad Nacional Agraria la Molina, Av. La Molina S/N, Lima 12, Perú, wvasquez@, lamolina.edu.pe
} 
the nutritional requirements of the fish. These four diets were supplied for two months and a commercial diet was used as a control. The fatty acid profile was determined for oils, diets and fillets, before and after the experiment. The levels of omega- 6 polyunsaturated fatty acids (n-6 PUFA) such as arachidonic acid (AA) and omega-3 polyunsaturated fatty acids (n-3 PUFA) such as eicosapentaenoic acid (EPA) and docosahexaenoic acid (DHA) were analyzed. The best PUFA profile in fillets $(2,11 ; 4,64$ and 7,0 \% AA; EPA and DHA respectively) was obtained with the diet elaborated with fish oil, however, the best vegetable oil that influenced the increase of PUFA profile in Piaractus brachypomus fillet $(3,11 ; 0,82$ and $3,71 \%$ of AA, EPA and DHA) was palm oil. It was demonstrated the ability to improve the PUFA profile in Piaractus brachypomus fillets.

Key words: Piaractus brachypomus, fatty acids, fillet, oil, palm, corn, soy, fish, diet.

\section{INTRODUCCIÓN}

En los últimos años el consumo de pescado de origen marino pelágico ha presentado un aumento a nivel mundial y la razón principal se basa en que es considerado como un alimento saludable, ya que aparte de ser buena fuente de proteínas, es reducido en calorías, grasa saturada y colesterol. Su amplia aceptación se fundamenta, principalmente, en que es un alimento rico en ácidos grasos poliinsaturados AGPI de la serie omega-3 (n-3), a los cuales se les atribuyen propiedades benéficas en cuanto a la disminución del riesgo de sufrir enfermedades cardiovasculares. Además de demostrar un efecto antiinflamatorio y citoprotector ${ }^{1}$.

Los peces marinos poseen niveles significativos de $\mathrm{AGPI}^{2}$. Mientras que la mayoría de peces de agua dulce, excluyendo a la trucha, contienen relativamente poco de los AGPIn-3. En este grupo se encuentra el paco Piaractus brachypomus una especie nativa de importancia económica en toda la Amazonía peruana. Sin embargo, la alimentación de los peces es determinante para el perfil de sus AGPI. Se ha demostrado que la composición de los ácidos grasos de los lípidos de la dieta influye en la composición de los ácidos grasos de los lípidos del músculo del pescado.

Estudios realizados determinaron el pobre aporte de AGPIn-3 de Piaractus brachypomus, constituyéndose en un problema nutricional ${ }^{3}$. Por otro lado, las especies de agua dulce aunque presenten una elevada concentración de AGPI del tipo omega-6 en comparación con los de la serie $n-3$, tienen la ventaja de presentar mayores concentraciones de AGPI de 18 carbonos

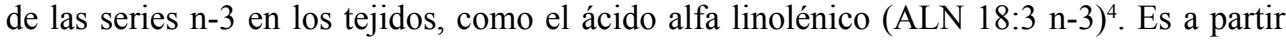
de esta ventaja, que estos peces de agua dulce tienen la habilidad de demostrar una mayor capacidad de elongar y desaturar el ácido alfa linolénico a ácido eicosapentanoico (EPA) y ácido docosahexanoico (DHA) ${ }^{5}$. Esta capacidad podría ser utilizada durante el proceso de cultivo, a fin de promover mediante una dieta, a mejorar el perfil de AGPI. 
Esto significa que muchas de estas especies pueden transformar un determinado ácido graso en su correspondiente de cadena más larga, con gran eficiencia, permitiendo que en la elaboración de dietas se incluyan aceites vegetales, siempre y cuando estos contengan cantidades adecuadas de ácido alfa linolénico (ALN), que pueden ser convertidos en EPA y DHA por el sistema enzimático del pez ${ }^{6}$, estas consideraciones justifican la necesidad de investigar en una de las principales especies amazónicas, el paco Piaractus brachypomus que es ampliamente demandado a nivel regional en la Amazonía y constituye significativamente uno de los principales alimentos proteicos, con la orientación de mejorar su perfil de AGPI saludable.

\section{PARTE EXPERIMENTAL}

\section{Lugar y duración de la evaluación}

El trabajo de investigación tuvo una duración de dos meses y se llevó a cabo en las instalaciones del centro de producción de la empresa Acuicultura y Pesquera Calicanto S.R.L ubicada en la ciudad de Pucallpa, provincia Coronel Portillo, región Ucayali.

\section{Evaluación de parámetros de producción}

Las unidades experimentales constaron de cinco corrales de $25 \mathrm{~m}^{2}$ instalados dentro de un estanque. Se trabajó con Piaractus brachypomus de $200 \pm 10$ g previamente seleccionados y distribuidos al azar en los corrales. Durante el experimento se realizaron biometrías quincenales y se evaluaron los principales parámetros de producción; tasa específica de crecimiento, tasa de crecimiento absoluto y relativo, factor de conversión del alimento (FCA), factor de condición (K) y supervivencia.

\section{Evaluación de parámetros de calidad de agua}

Los análisis físicos y químicos del agua de cultivo de los peces se realizaron semanalmente in situ utilizando un kit de análisis de agua HANNA instruments ${ }^{\circledR}$. El parámetro de temperatura se midió diariamente.

\section{Determinación de la composición química proximal de las dietas y filetes de Piaractus brachypomus}

Fueron utilizados los siguientes métodos de ensayo:

Proteína (Nx6,25); COVENIN 1195-1980 Alimentos. Determinación de Nitrógeno. Método Kjeldahl.

Grasa total; NTP 201.016 2002 Carne y productos cárnicos. Determinación del contenido de grasa total.

Humedad: NOM-116-SSA1-1994. Determinación de humedad en alimentos por tratamiento térmico. Método por arena o gasa.

Ceniza; NMX-F-607-NORMEX-2013 Alimentos - Determinación de Cenizas en Alimentos - Método de Prueba.

Fibra cruda; AOCS Official Method Ba 6-84. 6th Edition, Rev. Online. 2009 Crude Fiber by Procedure Using Glass Wool. 


\section{Determinación de la energía bruta en las dietas experimentales}

Se utilizó el método de energía total por bomba calorimétrica, mediante el principio de la oxidación total (combustión total) de una muestra de cada dieta en una bomba calorimétrica.

\section{Determinación del perfil de ácidos grasos poliinsaturados en las fuentes lipídicas y filetes de Piaractus brachypomus}

Antes de iniciar la alimentación con las cuatro dietas elaboradas y la dieta control, se tomaron tres muestras al azar de Piaractus brachypomus del lote proveniente para el experimento, y se determinó el perfil de AGPI para tener valores referenciales iniciales. De igual manera se determinó el perfil de AGPI a las fuentes lipídicas utilizadas en el experimento. Posteriormente después de iniciado el experimento se tomaron tres muestras de Piaractus brachypomus por cada corral, a los 30 y a los 60 días del experimento. Se aplicó la metodología AOCS Ce1b-89 Sixth Edition. 2009. Fatty Acid Composition of Marine Oils by GLC.

\section{Diseño experimental y diseño estadístico}

Diseño completamente al azar con cuatro tratamientos (dietas) y un tratamiento control. Cada tratamiento se llevó a cabo en corrales con una densidad de dos peces por metro cuadrado (20 peces por corral, instalados dentro de un estanque).

Para el tratamiento control se utilizó un alimento pelletizado comercial para peces amazónicos. La formulación de las cuatro dietas elaboradas contempló la misma cantidad y tipo de insumos proteicos y energéticos, con excepción en el tipo de fuente lipídica que fue objetivo de la presente investigación; aceites de palma, maíz, soja y pescado con una inclusión del $8 \%$.

\section{Variable evaluada}

La variable evaluada al inicio y a los 60 días de experimentación fue el perfil de AGPI omega-3 y omega-6 en los filetes de Piaractus brachypomus. Los resultados se sometieron a un análisis de varianza con un nivel de significancia de 0,05 y una prueba de comparación múltiple de medias de acuerdo con el criterio de Tuckey.

\section{RESULTADOS Y DISCUSIÓN}

\section{Parámetros de producción}

En la tabla 1 se muestran los resultados de los parámetros de producción obtenidos en el transcurso de dos meses de investigación. El FCA con valores entre 1,75 y 2,1 se consideran válidos en relación con los valores reportados de FCA $(3,34$ - 4,14) en juveniles de Piaractus mesopotamicus alimentados con tres niveles de proteína bruta $(18 \%, 22 \% \text { y } 26 \%)^{8}$. El factor de condición, generalmente designado como K, reflejó información acerca del estado fisiológico de los peces y del uso adecuado de las fuentes alimenticias y su valor en animales adultos no siempre refleja el estado alimenticio 9 . Con relación a los demás parámetros de crecimiento, como la tasa específica de crecimiento, fue importante tener en cuenta que este parámetro se vio influenciado no solo por el alimento, sino también por el estado fisiológico del pez, la densidad de crianza y la temperatura del agua ${ }^{9}$. 
Tabla 1. Parámetros de producción de Piaractus brachypomus, obtenidos durante el periodo de 60 días de alimentación con diferentes fuentes lipídicas.

\begin{tabular}{|c|c|c|c|c|c|}
\hline Parámetro & $\begin{array}{c}\text { Dieta } \\
\text { control } \\
\text { Alimento } \\
\text { comercial }\end{array}$ & $\begin{array}{c}\text { Dieta } 1 \\
\text { con aceite de } \\
\text { palma }\end{array}$ & $\begin{array}{c}\text { Dieta } 2 \\
\text { con aceite de } \\
\text { maíz }\end{array}$ & $\begin{array}{c}\text { Dieta } 3 \\
\text { con aceite de } \\
\text { soya }\end{array}$ & $\begin{array}{c}\text { Dieta } 4 \\
\text { con aceite de } \\
\text { pescado }\end{array}$ \\
\hline Peso inicial $(\mathrm{g})$ & $200 \pm 10$ & $200 \pm 10$ & $200 \pm 10$ & $200 \pm 10$ & $200 \pm 10$ \\
\hline Peso final (g) & $350 \pm 8$ & $345 \pm 9$ & $334 \pm 8$ & $340 \pm 8$ & $335 \pm 6$ \\
\hline Ganancia de peso $(\mathrm{g})$ & 150 & 145 & 134 & 140 & 135 \\
\hline Alimento consumido (g) & 262,5 & 261 & 268 & 259 & 283,5 \\
\hline $\begin{array}{l}\text { Factor de conversión del } \\
\text { alimento (FCA) }\end{array}$ & 1,75 & 1,8 & 2 & 1,85 & 2,1 \\
\hline Factor de condición, $\mathrm{K}$ & 2,53 & 2,59 & 2,71 & 2,52 & 2,58 \\
\hline Talla inicial $(\mathrm{cm})$ & $21 \pm 1,5$ & $21 \pm 1,5$ & $21 \pm 1,5$ & $21 \pm 1,5$ & $21 \pm 1,5$ \\
\hline Talla final $(\mathrm{cm})$ & $24 \pm 1,8$ & $23,7 \pm 1,9$ & $23,1 \pm 1,8$ & $23,8 \pm 1,8$ & $23,5 \pm 1,6$ \\
\hline $\begin{array}{l}\text { Tasa específica de } \\
\text { crecimiento (\%/día) }\end{array}$ & 1,67 & 1,67 & 1,67 & 1,67 & 1,67 \\
\hline $\begin{array}{l}\text { Tasa de crecimiento } \\
\text { absoluto (cm/día) }\end{array}$ & 0,05 & 0,05 & 0,04 & 0,05 & 0,04 \\
\hline $\begin{array}{l}\text { Tasa de crecimiento } \\
\text { relativo ( } \% / \text { día) }\end{array}$ & 0,24 & 0,21 & 0,17 & 0,22 & 0,20 \\
\hline
\end{tabular}

Tabla 2. Parámetros físico químicos de calidad de agua en la producción de Piaractus brachypomus.

\begin{tabular}{lc}
\hline Parámetro & Valor \\
\hline Temperatura ambiente $\left({ }^{\circ} \mathrm{C}\right)$ & $27,0 \pm 1.5$ \\
Temperatura agua $\left({ }^{\circ} \mathrm{C}\right)$ & $26,0 \pm 2$ \\
Oxígeno disuelto $(\mathrm{ppm})$ & $5,4 \pm 0.5$ \\
$\mathrm{pH}$ & $6,5 \pm 0.3$ \\
Amonio $(\mathrm{ppm})$ & 0,0 \\
Nitrito $(\mathrm{ppm})$ & 0,0 \\
Alcalinidad $(\mathrm{ppm})$ & $62,0 \pm 2$ \\
Dióxido carbono $(\mathrm{ppm})$ & $9,0 \pm 1$ \\
Dureza $(\mathrm{ppm})$ & $32,0 \pm 2.5$ \\
Conductividad eléctrica $(\mathrm{Us})$ & $319,0 \pm 5$ \\
Sólidos totales disueltos $(\mathrm{ppm})$ & $312,0 \pm 6$ \\
\hline
\end{tabular}

En la tabla 2, los parámetros de calidad de agua registrados para el cultivo de Piaractus brachypomus en el presente trabajo se encuentran dentro de lo adecuado ${ }^{9}$, sin embargo, la temperatura del agua de $26^{\circ} \mathrm{C}$ se encontró por debajo del rango de 28 a $30^{\circ} \mathrm{C}^{10}$. Esto se debe a que en el periodo cuando se realizó la investigación aún alcanzaba la estación más fría del año en la región Ucayali.

\section{Fuentes lipídicas y perfil de ácidos grasos poliinsaturados}


Uno de los ingredientes utilizados en la formulación de alimentos para peces fue el aceite de pescado que se utilizó para suministrar energía dietética y ácidos grasos esenciales; sin embargo, este insumo ha alcanzado la meseta de su producción, además que es un recurso finito $^{11}$. De los insumos mencionados en la tabla 3; los aceites de soya y maíz generalmente son destinados para consumo humano directo, de aquí se tomó en cuenta los costos al ser utilizados como insumo en la elaboración de alimento para peces. Un posible sustituto del aceite de pescado en los alimentos acuícolas es la palma, considerado el segundo aceite vegetal de mayor volumen producido en el mundo. Al respecto, se debe mencionar que el aceite crudo de palma es un insumo regional de la Amazonía, el cual debería ser estudiado con mayor énfasis considerando el aspecto económico.

Tabla 3. Parámetros físico químico de las fuentes lipídicas, en porcentaje.

\begin{tabular}{lcccc}
\hline \multicolumn{1}{c}{ Parámetro } & Aceite de palma & Aceite de maíz & Aceite de soya & Aceite de pescado \\
\hline Proteína & 0,03 & 0,03 & 0,00 & 0,14 \\
Grasa cruda & 99,61 & 99,90 & 99,84 & 99,96 \\
Humedad & 0,39 & 0,10 & 0,16 & 0,04 \\
Ceniza & 0,01 & 0,01 & 0,00 & 0,04 \\
Fibra cruda & 0,00 & 0,00 & 0,00 & 0,00 \\
Energía $(\mathrm{kcal} / \mathrm{kg})$ & 8965,02 & 8991,12 & 8985,60 & 8996,96 \\
\hline
\end{tabular}

En la tabla 6 se reporta el perfil de ácidos grasos de las fuentes lipídicas utilizadas para la elaboración de las dietas del presente experimento. El aceite de pescado fue el único insumo que aportó directamente ARA, EPA y DHA a la dieta, los demás insumos como el aceite de palma, soya y maíz no aportaron estos ácidos grasos. Sin embargo, sí contribuyeron con el aporte de ácidos grasos monoinsaturados como el ácido oleico y de ácidos grasos poliinsaturados como linoleico. El aceite de palma presentó una alta concentración de ácidos palmítico (saturado) y oleico (monoinsaturado) ${ }^{12}$, el aceite de palma utilizado en el presente trabajo reportó $39,59 \%$ y $41,70 \%$ correspondientemente.

El aceite de soya se utiliza principalmente para freír y cocinar, industrialmente forma parte de alimentos para humanos y animales ${ }^{12}$, presentó ácidos AGPI como el ácido linoleico y alfa linolénico en un porcentaje de $54 \%$ y 7,38\%, respectivamente; ácidos grasos monoinsaturados como el ácido oleico en un 20,96 \% y ácidos grasos saturados como el ácido palmítico en un $10,82 \%$.

Con respecto al aceite de maíz utilizado, presentó principalmente el AGPI linoleico en un porcentaje de 55,50\%; y ácidos grasos monoinsaturados como el ácido oleico en un 28,49\% y el ácido palmitoleico en un $11,64 \%$.

El aceite de pescado utilizado fue el único de los cuatro insumos que presentaron los AGPI; AA, EPA y DHA, en valores de 1,24 \%; 18,54 \% y 11,74 \%, respectivamente. 


\section{Dietas de engorde para Piaractus brachypomus y perfil de ácidos grasos}

Con las cuatro fuentes lipídicas se elaboraron cuatro dietas. En la tabla 4 se presentan los parámetros físicos químicos encontrados, los mismos que estuvieron alrededor del $30 \% \mathrm{y}$ $29,2 \%$ de proteína de acuerdo a los requerimientos de la especie ${ }^{9}$. Se recomienda un $30 \%$ de proteína para Piaractus brachypomus ${ }^{4}$. En este sentido, los valores de proteína en las dietas de experimentación cumplieron con los requerimientos de la especie.

Con respecto a los niveles de grasa, en las dietas elaboradas se superó el $4 \%$ a $6 \%{ }^{9}$, mientras que para la dieta comercial se mantuvo en el margen recomendado.

Los carbohidratos de las dietas sobresalen el valor de $36 \%{ }^{9}$. La cantidad de energía de las dietas superó ampliamente el requerimiento de $3410 \mathrm{kcal} / \mathrm{kg}^{13}$.

En la tabla 6 se muestra el perfil de ácidos grasos en cada dieta elaborada, destacó la dieta elaborada con aceite de palma que presentó la mayor proporción del ácido graso saturado palmítico $(32,85 \%)$, esto debido a que el aceite de palma es el insumo que más cantidad de ácido palmítico presentó $(39,59 \%)$ en relación a los demás aceites de maíz, soja y pescado $(0,0 \% ; 10,82 \%$ y $15,51 \%$, respectivamente). La dieta comercial fue la que menor proporción de AGPI presentó ( $0,00 \%$ de AA, $0,00 \%$ de EPA y $0,14 \%$ de DHA), mientras que la dieta elaborada con aceite de pescado fue la que mayor proporción de AGPI presentó $(0,95 \%$ de AA, $14,77 \%$ de EPA y $9,43 \%$ de DHA), esto se debe a que en la dieta comercial se encontró menor porcentaje de grasa $(3,60 \%)$.

Tabla 4. Parámetros físico químico de las dietas elaboradas con diferentes fuentes lipídicas, en porcentaje.

\begin{tabular}{lccccc}
\hline Parámetro & $\begin{array}{c}\text { Dieta control } \\
\text { Alimento } \\
\text { comercial }\end{array}$ & $\begin{array}{c}\text { Dieta 1 } \\
\text { con aceite de } \\
\text { palma }\end{array}$ & $\begin{array}{c}\text { Dieta 2 } \\
\text { con aceite de } \\
\text { maíz }\end{array}$ & $\begin{array}{c}\text { Dieta 3 } \\
\text { con aceite de } \\
\text { soya }\end{array}$ & $\begin{array}{c}\text { Dieta 4 } \\
\text { con aceite de } \\
\text { pescado }\end{array}$ \\
\hline Proteína & 31,01 & 31,19 & 29,83 & 30,96 & 29,90 \\
Grasa cruda & 3,60 & 13,71 & 13,81 & 14,18 & 13,86 \\
Humedad & 10,10 & 10,21 & 10,19 & 9,73 & 9,92 \\
Ceniza & 9,40 & 7,40 & 7,40 & 7,50 & 7,40 \\
Fibra cruda & 4,03 & 3,16 & 2,07 & 2,12 & 2,20 \\
CHOs & 41,86 & 34,33 & 36,70 & 35,51 & 36,72 \\
Energía (kcal/kg) & 3975,90 & 4611,60 & 4565,10 & 4497,30 & 4582,30 \\
\hline
\end{tabular}

\section{Filetes de Piaractus brachypomus y perfil de ácidos grasos}

En la tabla 5 se exponen los parámetros físico químico de los filetes de Piaractus brachypomus obtenidos después del periodo de experimentación, en todos los tratamientos los valores están dentro de los valores encontrados por el Instituto Tecnológico Pesquero del Perú; la proteína se encuentra dentro del rango de $15,7 \%$ a $18,7 \%{ }^{14}$. Piaractus brachypomus es considerado como una especie grasa (mayor al $5 \%$ de grasa) ${ }^{15}$. Esta especie presenta una gran fluctuación en el contenido graso de casi 31 veces, el contenido más alto fue observado en el mes de abril $(8,7 \%)$, y el más bajo en noviembre $(0,82 \%)^{14}$. 
Tabla 5. Parámetros físico químico, filetes de Piaractus brachypomus, en porcentaje

\begin{tabular}{lccccccc}
\hline Parámetro & $\begin{array}{c}\text { Inicio de } \\
\text { experimento }\end{array}$ & $\begin{array}{c}\text { Dieta } \\
\text { comercial }\end{array}$ & $\begin{array}{c}\text { Aceite } \\
\text { de } \\
\text { palma }\end{array}$ & $\begin{array}{c}\text { Aceite } \\
\text { de maíz }\end{array}$ & $\begin{array}{c}\text { Aceite } \\
\text { de soya }\end{array}$ & $\begin{array}{c}\text { Aceite de } \\
\text { pescado }\end{array}$ & ITP (2009) \\
\hline Proteína & 18,14 & 18,38 & 17,41 & 17,80 & 18,23 & 18,38 & $14,96-18,90$ \\
Grasa cruda & 1,30 & 2,50 & 2,30 & 5,30 & 3,80 & 2,80 & $2,73-18,09$ \\
Humedad & 79,44 & 77,60 & 78,50 & 75,92 & 76,47 & 77,90 & $64,29-79,12$ \\
Ceniza & 1,08 & 1,55 & 1,28 & 1,38 & 1,71 & 1,40 & $0,87-1,40$ \\
Fibra cruda & 0,00 & 0,00 & 0,00 & 0,00 & 0,00 & 0,00 & - \\
\hline
\end{tabular}

Según la tabla 6, para las dietas donde se utilizaron aceites vegetales de palma, maíz y soja, se determinaron valores de ácido araquidónico (AA) de 3,11\%; 1,25\% y 1,38 \%, correspondientemente en los filetes de Piaractus brachypomus luego del periodo de experimentación. La presencia de AA en estos filetes, considerando que en las fuentes lipídicas no existe representación de AA, se debe al proceso de bioconversión del ácido linoleico, que comprende elongaciones (incorporación de dos carbonos) y desaturaciones (incorporación de un doble enlace) acometidas sucesivamente por las $\Delta 6, \Delta 5$ y $\Delta 4$ desaturasas ${ }^{16}$. En este caso, los aceites de palma, maíz y soja aportaron niveles de ácido linoleico del orden de 10,52 \%; $55,50 \%$ y 54,04 \%, respectivamente, que por el proceso de bioconversión se transformaron en AA. Por otro lado, en los filetes de Piaractus brachypomus alimentados con dieta comercial, con aceite de palma, maíz, soja y pescado se determinaron valores de $16,20 \%$; 14,26 \%; $23,04 \% ; 21,28 \%$ y 13,11\% de ácido linoleico. Peces de agua dulce reportaron niveles entre $13,77 \%$ y $26,24 \%{ }^{17}$.

Para el caso del ácido alfa linolénico en estudios realizados se encontraron niveles entre $0,25 \%$ a $1,53 \% 17$ y Andrade et al. ${ }^{17}$ encontraron valores inferiores al 2,50 \%, y en algunas especies no fue detectado.

El mayor porcentaje del ácido linoleico sobre el ácido alfa linolénico es un común denominador en la mayoría de las especies de agua dulce, incluido el Piaractus brachypomus, y esto debido a los hábitos alimenticios o al tipo de alimentación en los estanques de cultivo. Por lo tanto, la relación que puede existir entre estos dos importantes ácidos grasos puede ser direccionada según el objetivo de crianza ${ }^{17}$.

Los estudios realizados por el Instituto Tecnológico Pesquero del Perú ${ }^{14}$ demostraron entre ciertos ácidos grasos, un valor de 0,318 \% para el DHA. Se sabe que la composición de ácidos grasos de los filetes de pescado refleja la composición de ácidos grasos del aceite usado en la dieta ${ }^{11}$. Teniendo en cuenta este enunciado y en relación a los AGPI AA, EPA y DHA, el aceite de pescado es la única fuente lipídica que aportó directamente estos tres AGPI, como se puede observar en la tabla 6. Sin embargo, al comparar con el aporte de AGPI de las dietas, se observó que en las dietas elaboradas con aceite de palma, maíz y soja, 
se tuvo presencia de EPA en valores de $2,64 \% ; 2,42 \%$ y $2,44 \%$, respectivamente, así como DHA en valores de $1,55 \% ; 1,40 \%$ y $1,46 \%$, respectivamente. La presencia de estos AGPI en estas dietas se debió a la harina de pescado que aporta estos AGPI.

Mientras que en la dieta con aceite de pescado, el nivel alcanzado de EPA y DHA alcanzó valores de $14,77 \%$ y $9,43 \%$, correspondientemente, esto se debería por la presencia de harina y aceite de pescado como ingredientes, que amplían el nivel de EPA y DHA. Por otro lado, se debe considerar que estos peces de agua dulce tienen la habilidad de demostrar una mayor capacidad de elongar y desaturar el ácido alfa linolénico a EPA y DHA ${ }^{16}$.

Según estudios realizados ${ }^{17}$ se determinó el pobre aporte de AGPIn-3 de Piaractus brachypomus, constituyéndose en un problema nutricional desde el aspecto de aporte de AGPIn-3. Sin embargo, con el presente estudio, se demostró la capacidad de mejorar el perfil de AGPI durante el proceso de cultivo. Lo cual constituye un indicador de la calidad nutricional de esta especie y de la posibilidad de considerarlo como un alimento funcional.

Los peces de agua dulce pueden ser usados como una dieta saludable para humanos, considerando que su calidad nutricional es aún mejor, ya que además de tener considerables cantidades de APGI n-3, los peces de agua dulce contienen mayores niveles de ácido araquidónico que los peces marinos ${ }^{2}$. 


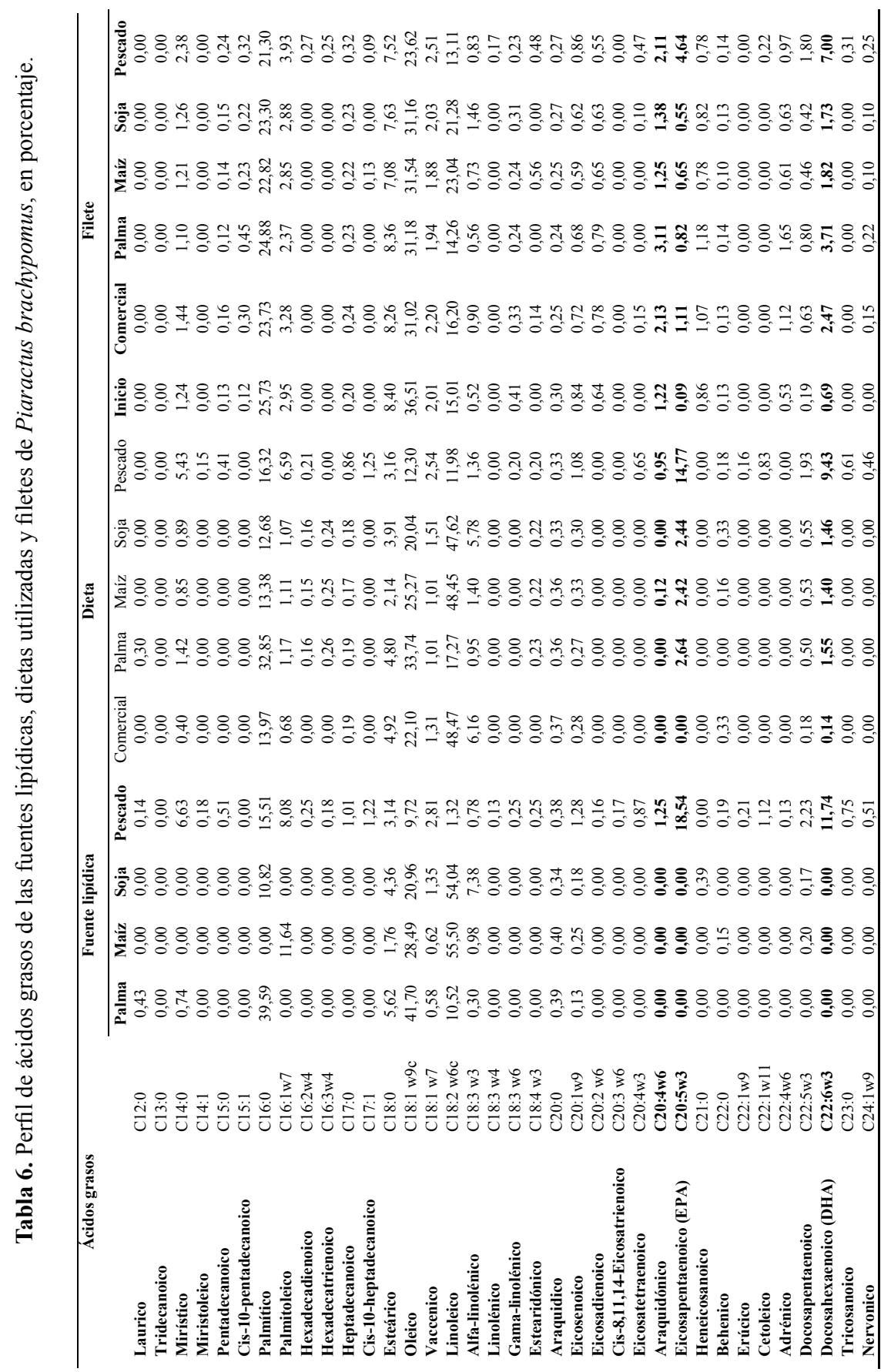




\section{CONCLUSIONES}

En consecuencia, a lo realizado en el presente trabajo de investigación y en función a los resultados obtenidos, se puede concluir que:

La fuente lipídica de origen vegetal, que mejor influye en el incremento de ácidos grasos omega-3 en filete de paco Piaractus brachypomus fue el aceite de palma.

La dieta que presentó mejor perfil de ácidos grasos fue la preparada con aceite de pescado, seguida de la dieta elaborada con aceite de palma.

El perfil de ácidos grasos poliinsaturados en filetes de paco Piaractus brachypomus fue mejorado con la inclusión de fuentes lipídicas de origen vegetales como el aceite de palma en un $8 \%$.

Se demostró la capacidad de mejorar el perfil de ácidos grasos poliinsaturados durante el proceso de cultivo de paco Piaractus brachypomus.

\section{AGRADECIMIENTO}

Los autores agradecen por el apoyo brindado a la presente investigación a las empresas: Acuicultura y Pesquera Calicanto S.R.L ubicada en la ciudad de Pucallpa, provincia Coronel Portillo, región Ucayali.

\section{REFERENCIAS BIBLIOGRÁFICAS}

1. Valenzuela R, Tapia G, González M y Valenzuela A. Ácidos grasos omega-3 (EPA y DHA) y su aplicación en diversas situaciones clínicas. Rev Chil Nut. 2011; 38(3): 356367.

2. Steffens W. Effects of variation feeds on nutritive in essential fatty acids in fish value of freshwater fish for humans. Aquaculture. 1997; 151: 97-119.

3. Perea A, Gomez E, Mayorga Y, y Triana C. Caracterización nutricional de pescados de producción y consumo regional en Bucaramanga. Arch Lat Nut. 2008; 58(1): 91-97.

4. FAO. Programa Cooperativo Gubernamental: Nutrición y alimentación de peces y camarones cultivados. Documento de campo No 4, Programa AQUILA I. Brasilia: FAO; 1989.

5. Moreno, J. Cambios en el perfil de ácidos grasos de filete de tilapia nilótica Oreochromis niloticus en respuesta a diferentes fuentes lipídicas [Tesis Magister en Producción Animal]. Bogotá, Colombia: Universidad Nacional de Colombia; 2016. [Citado el 20 jun 2016]. Disponible en http://www.bdigital.unal.edu.co/39433/1/ jennymarcelamorenopoveda.2013.pdf. 
6. Turchini G, Francis D, De Silva S. Fatty acid metabolism in the freshwater fish Murray cod (Maccullochella peelii peelii) deduced by the whole body fatty acid balance method. Comp Biochem Physiol B Biochem Mol Biol. 2006; 144(1): 110-118

7. OTCA, Organización del Tratado de Cooperación Amazónica. Piscicultura amazónica con especies nativas. Lima: Secretaria Pro- Tempore; 2016.

8. Fernandes J; Carneiro D; Sakomura N. Fontes e Níveis de Proteína Bruta em Dietas para Alevinos de Pacu (Piaractus mesopotamicus). R Bras Zootec. 2000; 29(3): 646-653.

9. Salinas A. Niveles de energía y relación proteína a energía sobre el desempeño productivo y composición corporal del paco (Piaractus brachypomus). [Tesis Mg Sc. en Nutrición]. Lima, Perú: Universidad Nacional Agraria La Molina; 2018.

10. Gomes F. Desempenho do tambaqui (Colossoma macropomum), da pirapitinga (piaractus brachypomum), e do híbrido tambatinga ( $C$, macropomum xp, brachypomum) mantidos em viveiros fertilizados na fase de engorda, Dissertação [Tesis de maestria]. Goias, Brasil: Universidad Federal de Goías; 2009.

11. Wing-Keong. Potential of palm oil utilisation in aquaculture feeds. Asia Pacific J Clin Nutr. 2000; 473-S476

12. Durand S, Torres J, y Sanhueza J. Aceites vegetales de uso frecuente en Sudamérica: características y propiedades. Nut Hosp. 2015; 32(1): 11-19.

13. Miranda D. Digestibilidad de ingredientes y determinación del requerimiento de energía digestible de paco (Piaractus brachypomus). [Tesis Mg Sc. en Nutrición]. Lima, Perú: Universidad Nacional Agraria La Molina; 2018.

14. ITP. Instituto Tecnológico Pesquero del Perú. Información nutricional sobre algunos peces comerciales de la Amazonía Peruana, Boletín de investigación. Lima, Perú. 2009.

15. Cortez J. Características bromatológicas de dieciséis especies hidrobiológicas de la Amazonía peruana en época de creciente. Folia Amazónica. 1992; 4(1): 111:118.

16. Guillaume J, kaushik S, Bergot $\mathrm{P}$, y Métailler R .Nutrición y alimentación de peces y crustáceos. Edición Mundi-Prensa, España. 2004.

17. Tueroz G. Comportamiento productivo y composición lipídica del paco (Piaractus brachypomus) alimentado con diferentes relaciones de ácidos grasos omega 6 a 3. [Tesis Mg Sc. en Nutrición]. Lima, Perú. Universidad Nacional Agraria La Molina. 2019. 